\title{
Design and Measurement of BER of faded QPSK modulated system
}

\author{
Arvinder Singh \\ Department of Computer Science Engineering \\ ASRA College of Engineering \&Technology, Bhawanigarh, India \\ Rajnish Kansal \\ Faculty of Computer Science Engineering Department \\ ASRA College of Engineering \&Technology, Bhawanigarh, India
}

\begin{abstract}
Wireless spectrum is considered a limited and valuable communications resource as it influences productivity, security and our daily lives. One of the driving strengths of QPSK using Gaussian method is the Signal-to-Noise Ratio (SNR) estimation and feedback channel for adaptation. Sudden time varying channel degrading effects sometimes require the transmission link to react appropriately so it can minimize the Bit Error Rate (BER). Thus, the objective of the thesis introduces a QPSK modulated scheme which utilizes a simple moments based SNR estimator for spectral and quality improvement for the wireless channel. Results of the our proposed method shows that Bit error rate in our proposed QPSK using Gaussian method modulated channel decreases as compared to the QPSK modulated channel.
\end{abstract}

Keywords - QPSK, BER, Wireless, Channel.

\section{INTRODUCTION}

The development of wireless technologies has significantly changed our way of communicating and sharing information [1]. Easy access to networks is available at any time, and at any location, as long as we can build wireless connections among devices that are capable of transmitting and receiving signals in the open air. Wireless devices are usually allowed to move, while wireless connections can still be set up and maintained. Movement of devices and other objects in the propagation medium introduces dynamics in the connection quality of a wireless network. The connectivity may vary over time due to change of device location, moving speed, or time-varying interference in the area, etc.

The wireless dynamics challenge both wireless protocol design and evaluation. To build and maintain network connectivity on unreliable and hard-to-control wireless connections, protocols need to estimate, predict, and adapt to variations. On the other hand, performing controlled and realistic evaluation is also challenging because the wireless environment changes unpredictably and uncontrollably over time in the wild test-beds, while physical world properties are usually not reproduced accurately in simulation and emulation platforms[2].

\section{DIGITAL MODULATIONS}

Modulation [3] is the process of changing the amplitude, frequency or phase of a radio frequency carrier signal (a carrier) to change with the information signal (such as voice or data). Four modulations are supported by the IEEE 802.16 standard: BPSK, QPSK, 16-QAM and 64-QAM. In this case the modulations used in the OFDM and OFDMA physical layers are introduced with a short description for each.

As for all modern communication systems, WiMax/802.16 uses digital modulation. The now well-known principle of a digital modulation is to modulate an analogue signal with a digital sequence in order to transfer this digital sequence over a given medium, fiber, radio link, etc. (see Figure 1). This has great advantages with regard to classical analogue modulation: better resistance to noise, use of high-performance digital communication and coding algorithms, etc. 


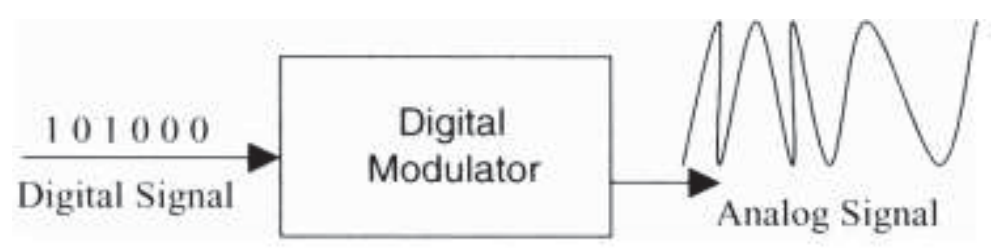

Figure 1. Digital modulation principles

\subsubsection{Binary Phase Shift Keying (BPSK)}

The BPSK is a binary digital modulation process; i.e. one modulation symbol is one bit. This gives high immunity against noise and interference and an extremely robust modulation. A digital phase modulation, which is the case for BPSK modulation, uses phase variation to encode bits: each modulation symbol is equivalent to one phase. The phase of the BPSK modulated signal is $\pi$ or $-\pi$ according to the value of the data bit. A frequently used illustration for digital modulation is the constellation. Figure 2 shows the BPSK constellation; the values that the signal phase can take are 0 or $\pi$.

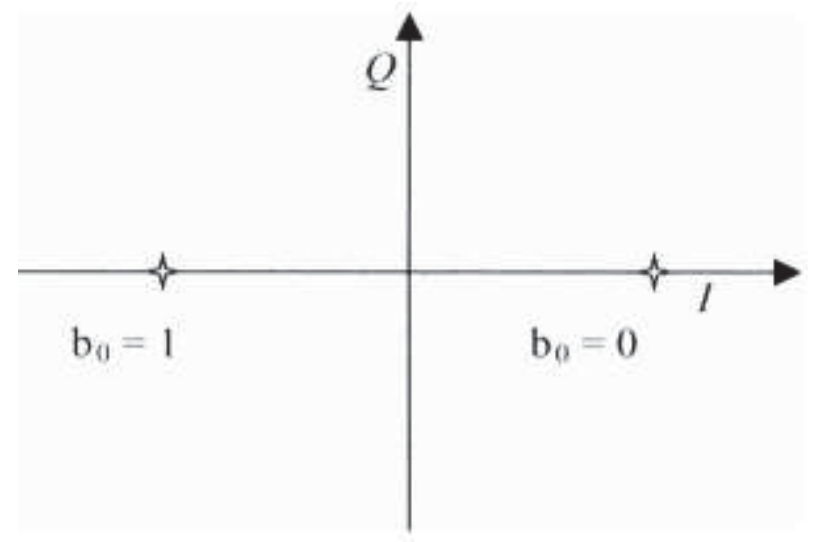

Figure 2. The BPSK

\section{Quadrature Phase Shift Keying (QPSK)}

When a higher spectral efficiency modulation is needed, i.e. more $\mathrm{b} / \mathrm{s} / \mathrm{Hz}$, greater modulation symbols can be used. For example, QPSK considers two-bit modulation symbols. QPSK is a type of modulation that uses 4 different phase shifts of a radio carrier signal to represent the digital information signal. These shifts are represented typically as +/- 45 and +/- 135 degrees. Many variants of QPSK can be used but QPSK always has a four-point constellation (see Figure 3).

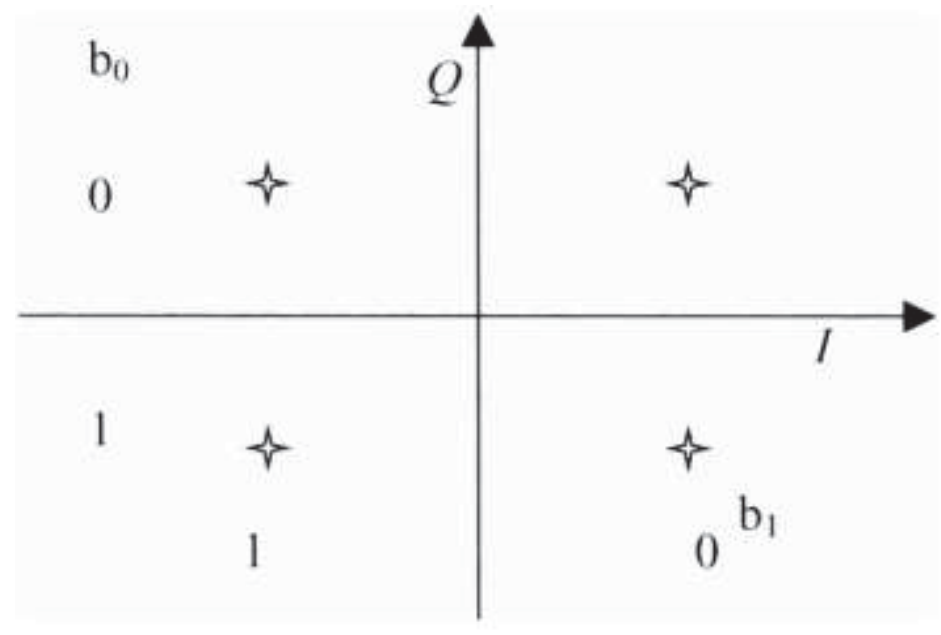

Figure 3. Example of a QPSK constellation 
The decision at the receiver, e.g. between symbol ' 00 ' and symbol ' 01 ', is less easy than a decision between ' 0 ' and ' 1 '. The QPSK modulation is therefore less noise resistant than BPSK as it has a smaller immunity against interference. A well-known digital communication principle must be kept in mind: 'A greater data symbol modulation is more spectrum efficient but also less robust.'

Quadrature Amplitude Modulation (QAM): 16-QAM and 64-QAM

QAM is a combination of amplitude modulation[4] (changing the amplitude or voltage of a sine wave to convey information) together with phase modulation. The QAM changes the amplitudes of two sinusoidal carriers depending on the digital sequence that have got to be transmitted; the two carriers being out of phase of $+\pi / 2$, this amplitude modulation is called quadrature. It should be mentioned that according to digital communication theory, QAM-4 and QPSK are the same modulation (considering complex data symbols). Both 16-QAM (4 bits/modulation symbol) and 64-QAM (6 bits/modulation symbol) modulations are included in the IEEE 802.16 standard.

\section{RESULTS}

In this case, simulation of multiple-input multiple-output (MIMO) multipath fading channels has been implemented. Two transmit antennas and two receive antennas are used with Rayleigh fading channel.

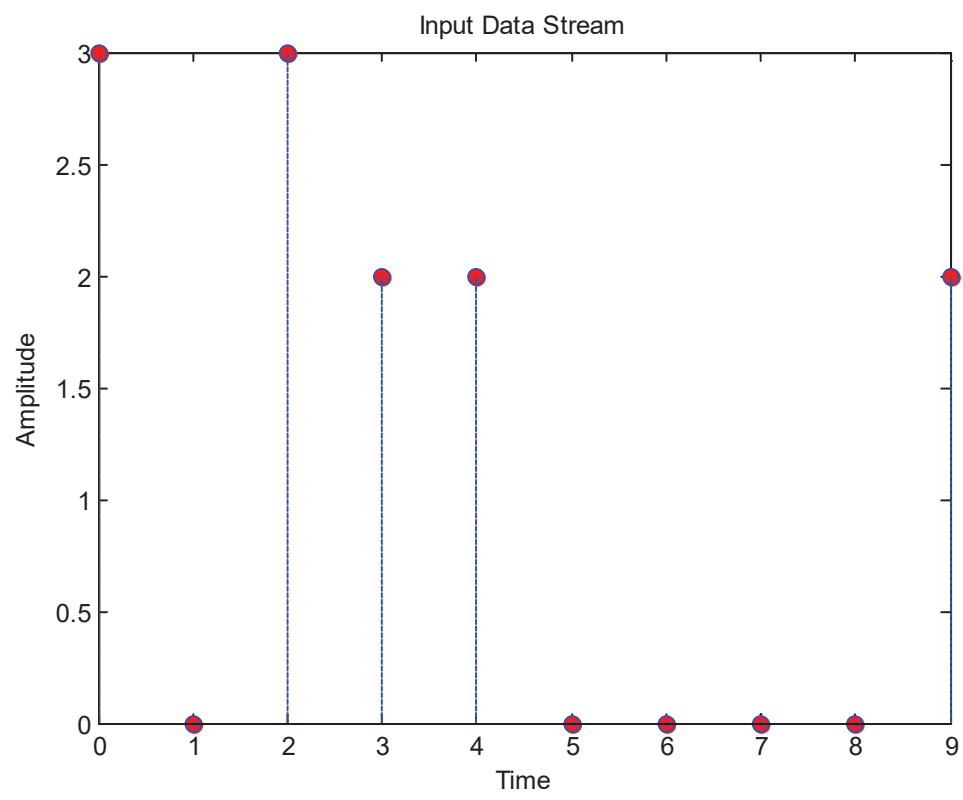

Figure 4. Input Data stream

Figure 4 shows the input data stream. 


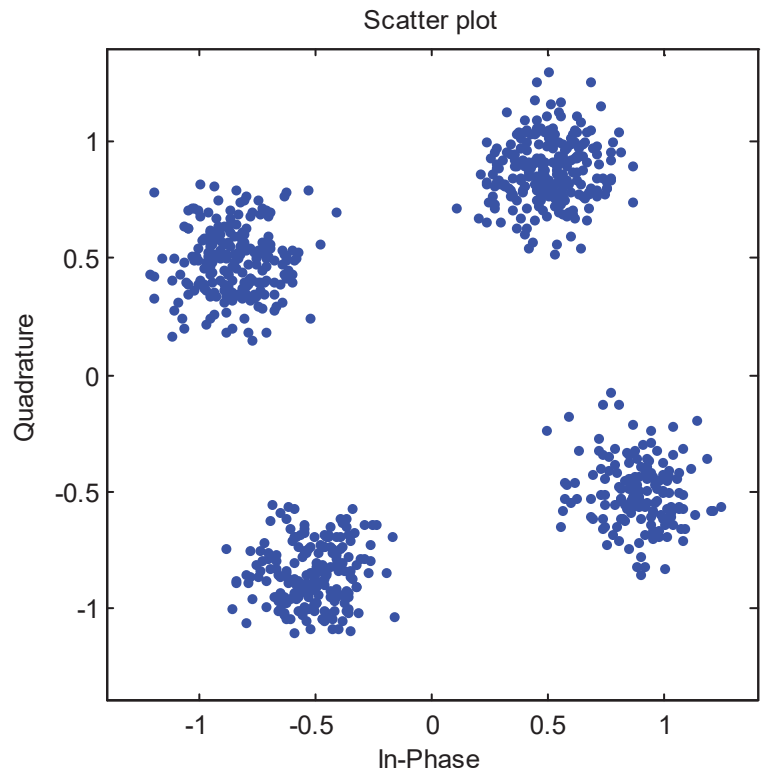

Figure 5. Scatter plot of the signal

Figure 5 shows the scatter plot of the signal.
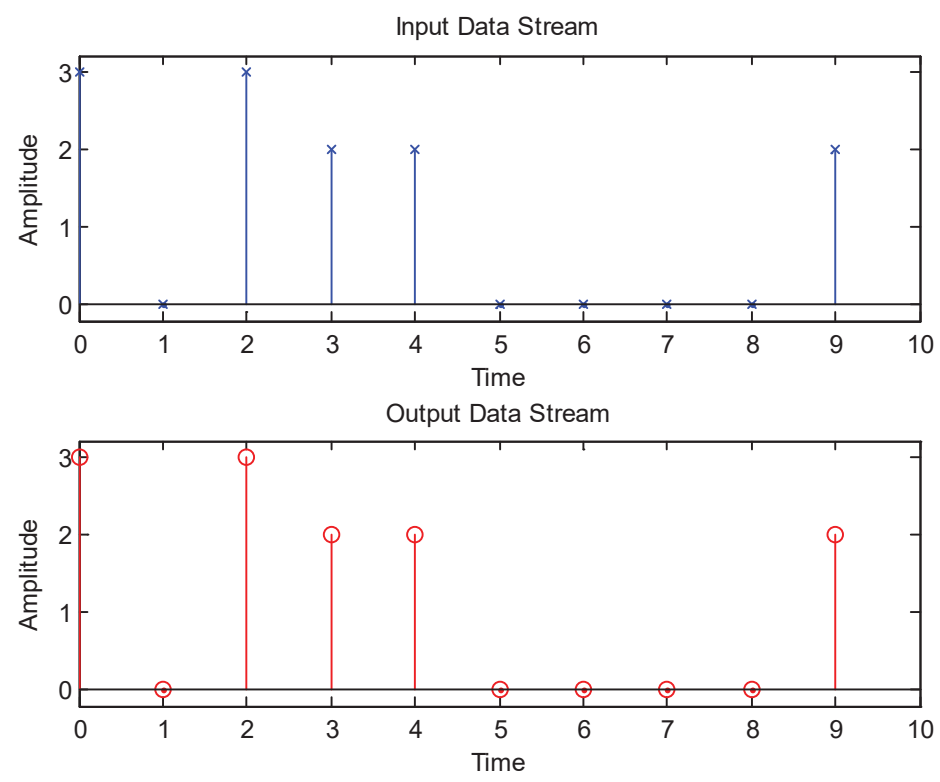

Figure 6. Input and output data stream

Figure 6 shows the input and output data stream. 


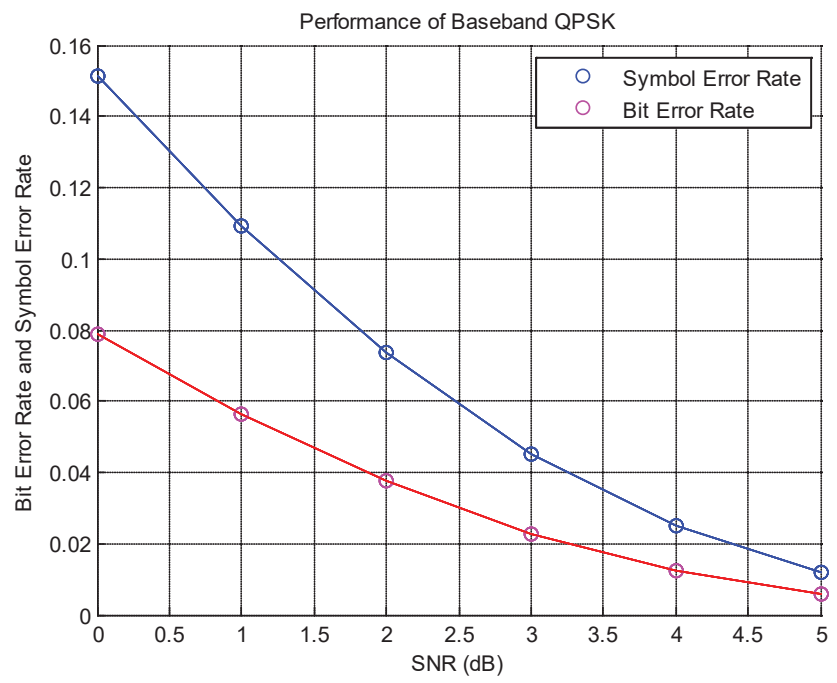

Figure 7. Bit error rate and symbol error rate versus signal to noise ratio

Figure 7 shows the bit error rate and symbol error rate decreases as the signal to noise ratio increases.

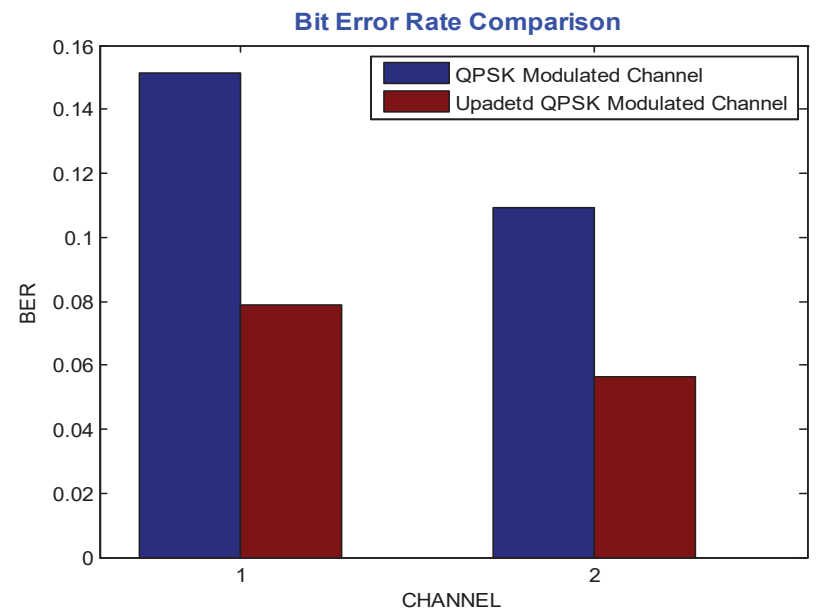

Figure 8. Comparison bar graph between QPSK and Updated QPSK Modulated Channel

Figure 8 shows the comparison between QPSK and Updated QPSK Modulated channel with Gaussian method for two channels. This shows that Bit error rate decreases in updated QPSK modulated channel as compared to the QPSK modulated channel.

As it known, wireless channels key problem is fading. In order to combat this fading and improve the capacity and the throughput of the system, multiple antennas at both ends of the communication link are used. This case analyzes the enhancement achieved with such structures, making a comparison of the performance of not only systems with multiple transmit but also multiple receive antennas.

\section{IV.CONCLUSION}

Wireless Communication is an application of science and technology that has come to be vital for modern existence. From the early radio and telephone to current devices such as mobile phones and laptops, accessing the global network has become the most essential and indispensable part of our lifestyle. Wireless communication is an everdeveloping field, and the future holds many possibilities in this area. Deployment of Wireless LAN increases well around the globe, it is increasingly important for us to understand different technologies and select the most appropriate one. MIMO channel also has been implemented and the throughput of the system has been calculated. As it known, wireless channels key problem is fading, in order to combat this fading and improve the capacity and the throughput of the system, multiple antennas at both ends of the communication link are used. This thesis analysed the performance analysis of MIMO in Rayleigh fading envelope and helps in analysing the security and 
data rate of the channel. Results of the our proposed method shows that Bit error rate in our proposed QPSK using Gaussian method modulated channel decreases as compared to the QPSK modulated channel.

\section{REFERENCES}

[1] Xiaohui Wang (2014), "Environment Models for Realistic Simulation and Emulation of Wireless Networks".

[2] Vijay Chandramouli, "A Detailed Study on Wireless LAN Technologies".

[3] Shanar H. Askar(2010), "Performance Evaluation of Fixed WiMax Physical Layer under High Fading Channels".

[4] https://en.wikipedia.org/wiki/Phase-shift_keying. 